\title{
Mobile Urban Drama - Setting the Stage with Location Based Technologies
}

\author{
Frank Allan Hansen, Karen Johanne Kortbek, and Kaj Grønbæk \\ Center for Interactive Spaces, \\ Department of Computer Science, University of Aarhus, \\ Aabogade 34, DK-8200, Aarhus N, Denmark \\ \{fah, kortbek, kgronbak\}@daimi.au.dk
}

\begin{abstract}
This paper introduces the novel concept of location-based Mobile Urban Dramas. In a Mobile Urban Drama the user become the main character in a play where actors' voices appear in the mobile phone headset linked to the physical setting in the city as the stage for the drama. The paper describes the dramaturgical concept and introduces a software framework supporting drama writers in developing such Mobile Urban Dramas. Experiences with use of the framework are discussed with successful examples of real dramas that have been developed and performed by a Danish theatre group, Katapult.
\end{abstract}

Keywords: Mobile drama, location-based technology, Semacodes, interactive audio, sensor/actuator based interaction, urban computing.

\section{Introduction}

Anna: "If you can do this, you will see me one more time. However, if you fail, you will end up talking to me for the rest of your life."

Christian: "You can't sit there and threaten me."

Anna: "My condolences. Goodbye."

Christian: "Anna?... Anna?!"

(Pause)

Narrator: "You must go and take a picture of tag three. It is hanging at the entrance of the pub."

(The end of scene 2 in the play GAMA).

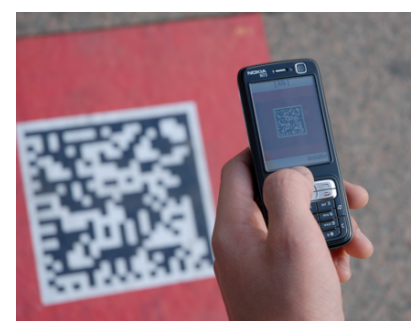

Fig. 1. Tags anchor scenes in the city

Mobile Urban Dramas are interactive audio plays that let the spectator (hereafter called 'user') be the main character in a drama where the real urban environment becomes the scenography. In the play, users are equipped with mobile phones, headsets and maps, and experience a drama in the streets of a city, where they trigger different scenes of the play through location-based technology such as GPS or Semacode ${ }^{1}$ based tags. Furthermore, they receive what appears to be SMS messages and phone calls, and encounter real life actors as part of the play that takes place along a pre-specified, possibly branching path through a cityscape or landscape. The

\footnotetext{
${ }^{1} \mathrm{http}: / /$ semacode.com/
} 
Danish theatre company Katapult [10] has developed three such plays using our technology. Katapult has developed these plays based on what they call an "AudioMove" concept. The AudioMove concept is structured as a single user experience, while the Mobile Urban Drama concept presented in this paper can be both single and multi user experiences. These plays are to our knowledge among the world's first applications of mobile phones for location-based theatre/drama.

Only few previous examples of using phones for drama exist. The most prominent example launched in 2005 by the German theatre collective Rimini Protokoll, as the world's first mobile phone theatre. The play was entitled "Call Cutta" [15] and took place in Kolkata, Calcutta, India. The play transforms the city into a stage and the people in the audience into main characters of the play. When the users buy a ticket at the box office, they may get the key for a room and a sketch of how to get there. It may be an arbitrary room in the urban space, e.g. an office, or an apartment. When entering the room they find a phone ringing, and the play then goes on with a call center person and the users as the main actors of the play, and the plot develops through the phone conversation. The room is the scenography; TVs or computers may turn on and integrate in the play. The play is today (2008) called "Call Cutta in a Box" and can be experienced in several cities around the world.

Rimini Protokoll are also known for a different mobile play "Cargo Sofia" [4], where the scenes take place in a Bulgarian truck driving in the streets of Dublin. Here the users are loaded into the container of a cargo truck, and they experience the drive through a video projection with audio on the container wall coming from the cab showing small film clips mixed with dialogue between the two drivers (actors). The users experience projections on the window, audio scenes in earbuds, and "meet" actors in the street when the truck appears at specific locations.

A final example of a mobile drama is the mobile TV project Song fuer C [19], which is a mobile detective story developed by Marc Weis and Martin DeMattia, Vodafone R\&D, and the Hochschule für Gestalung und Kunst Zürich (HGKZ). The user experiences video scenes on a Sony PSP or a mobile phone, while being guided around in some urban area where real actors may appear at certain locations.

For traditional computers, dramas that make the user into the main character are well known. One of the typical examples is Façade [12, 13]. It is a real-time, firstperson dramatic world in which the player, visiting a married couple in their apartment, becomes entangled in the conflict dissolution of their marriage. The Façade interactive drama integrates real-time autonomous agents, and AI is applied [6] to manage the behavior of agents actors, thus the user never experience real or recorded human characters in this type of play.

This paper discusses the new genre of Mobile Urban Drama. The paper is structured as follows: Section 2 introduces the location based framework for managing the plot graph and the current implementation. Section 3 discusses three example plays and the initial experiences. Section 4 compares our concept to related work. Section 5 concludes the paper.

\section{Setting the Stage with Location Based Technology}

This section presents our conceptual framework for Mobile Urban Drama. The framework draws on elements from interactive storytelling $[12,17,20]$, hyperfiction 
[3], adaptive hypertext [5], and user modeling [11] in order to create a rich model for storytelling. We also discuss how to realize the framework by applying technologies that allow use of real physical locations as scenography for the dramas. The novelty of the work presented here, is the combination of classical techniques such as plot graphs for representing the narrative flow, user models for representing the user interaction and environment models for representing the physical environment and physical interaction through various input and output systems. The work thus builds on concepts from interactive storytelling [16, 17], urban storytelling $[2,14]$, and augmented reality fiction [18], but generalizes and integrates these concepts in order to create a tighter coupling between the urban scenography and the narrative and allow the interaction to be mediated by a combination of different pervasive technologies.

\subsection{A Conceptual Framework for Mobile Urban Drama}

In the conceptual framework, dramas are constructed as a series of events that form the story. These events can be depicted as a plot graph inspired by Ryan [17] as illustrated in Figure 2.
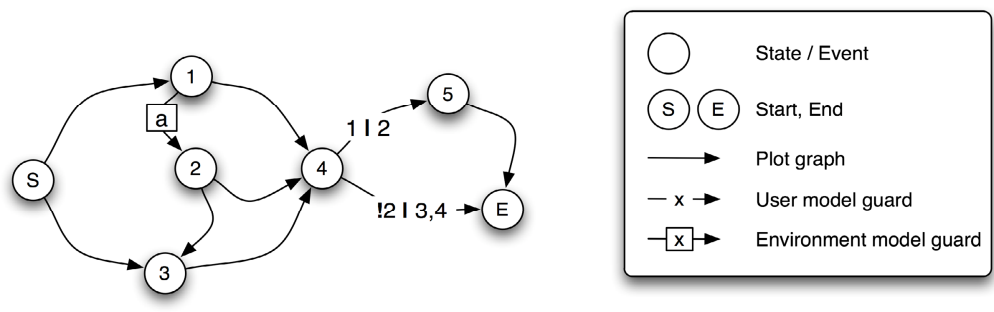

Fig. 2. A plot graph illustrating the basic elements of the framework. The plot is represented as a directed graph with guards that control possible paths. The guards specify node names (e.g. 1), sequences (e.g. 3,4), negations (e.g. !2), and / or values betweens these (e.g. 1 I 2).

The plot graph is a directed graph with nodes corresponding to events and edges specifying the valid ordering of the events. The plot graph must contain one or more start (S) and end events (E), where the story can begin and end, and any number of edges between the event nodes. The drama can be constructed as anything from a linear path through the nodes without any branches to a highly connected graph where the user freely can chose the next branch at each node. The graph can thus easily represent various interactive story architectures [17], e.g. trees that represent multiple variants of a story over time, flowcharts that are suitable for stories that contain episodes or levels such as games, and mazes, which represents hypertext like structures, where the plot is constructed from the users' actions at every node.

The basic plot graph only allows the author to specify events of the drama and their ordering. The author has no control of how the user actual follows the events. If the graph e.g. contains a loop, the user may experience the same event more than once and may in fact choose to follow the loop-sequences infinitely. To give the author more control of the plot, the framework includes two types of models, a user model and an environment model, which can be used in conjunction with the plot graph. 
The user model is an overlay model [5] on the plot graph and describes the user's knowledge of the story. The model encodes the events the user has experienced, the ordering of the events and the choices taken at different branches in the story. Based on the user's current user model, the author can add guards (conditions) to the edges in the plot graph that controls whether or not that specific path will be available to the user. In this way the user's actions can have consequences at a later state in the story and the author can control the users possibilities e.g. based on the persons, the user has met or the places she has visited in the virtual world. As an example, consider Figure 2 above: the user may follow different paths in order to get to the event labeled 4. If the user has visited state 1 or 2 , then she has the possibility to go through state 5 before ending the story, but if she has not experienced state 2 or has gone through state 3 and 4, the author sends her straight to the end in state E. In, this example the two paths are mutually exclusive, so based on the user's previous actions in start node $S$ and node 1, the author controls the path the user must follow in the end.

The second model, the environment model, has a similar function as the user model, but is not directly controlled by the user's actions. The environment model is used to describe the physical environment of the play, e.g. at which physical locations events occur, what technologies are used to trigger the events, and so forth. The environment model also captures the global state of the play. For instance, if the drama supports multiple users participating in a play at the same time, some of their actions may be fed back to the environment model, and the choice of one user may influence the interaction space of the others. In Figure 2, an environment model guard is placed between state 1 and state 2 . If one user has chosen to follow path from state 1 to state 2, the author could use the guard to make it less likely that this path would be available to other users at a later time of the play.

\subsection{Technical Realisations of the Conceptual Framework}

The conceptual framework discussed above only describes the interactive elements at the story level, but not how the drama is actually realized and integrated with real world scenography. However, the mapping between the conceptual model and the actual implementation is rather simple in that each story event (plot graph node), is triggered by a real event in the implementation. Events are triggered by some input from the user or a sensory system and the story event is provided by some kind of output to the user, as illustrated in Figure 3.

Inputs are used to trigger story events in the drama. Inputs may be anything from manual entry by the user, e.g. pressing a button on a mobile phone, to more automated input from a sensor, e.g. Semacodes, RFID, GPS, Bluetooth, or WiFi positioning systems. In terms of our system, it makes no difference how the input is generated, as long as it can be mapped to unique story events. However, the input technology will influence the interaction between the user and the real world scenography, and thus may impact the overall experience. Some technologies such as Semacode tags or RFID tags require the user to actively find and scan the tag either with the mobile phone's camera or an RFID scanner and the user must thus explicitly trigger the input.

Semacode tags are 2D patterns that encode a piece of text, typically an ID or a URL and can be used by software for camera phones. Such 2D barcodes can be used as digital-physical links. They have the advantage that they clearly visualize the link 
in the physical world, and when decoded, provide the link address directly (i.e. the URL). Semacodes have been used in a number of pervasive applications [7]. Other technologies like GPS can be used to support implicit interaction, as the system can register when a user enters a certain location and use this to generate input events [9]. In this case the user will not have to explicitly generate the event, but simply move about in the physical world as part of the play.
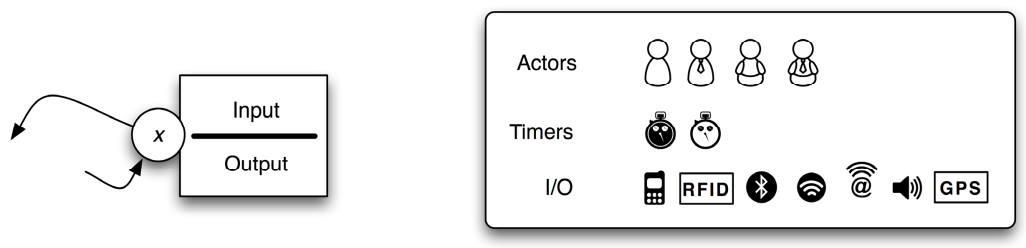

Fig. 3. Applying technology to the plot graph

The output from the system can also use various technologies to optimize the experience. If mobile phones are used as interaction devices in the mobile drama, the phone can play audio and display text, graphics, and film clips on the screen and thus function as a versatile output device. However, the physical environment may also be used to present output. Displays in the user's vicinity can show interactive graphics and (directional) speakers can provide audio or ambient effects. More permanent installations may also include 4D theater effects, such as wind, mist, or motion [1].

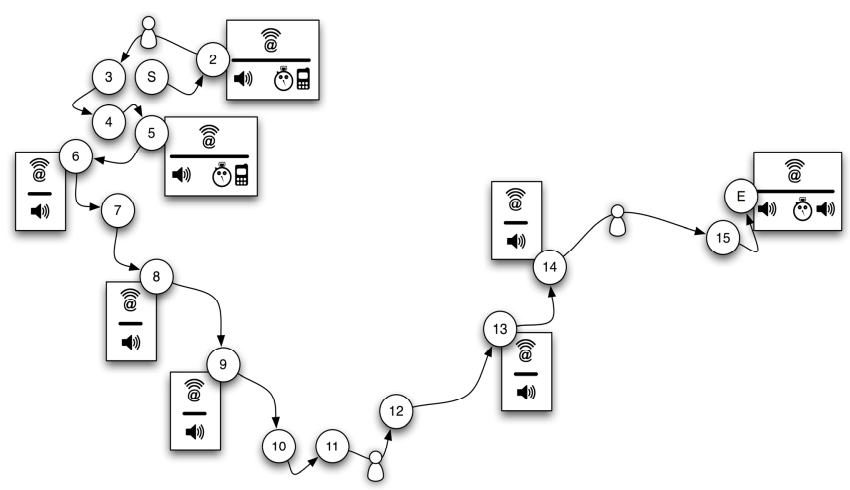

Fig. 4. Technical implementation of CORRIDOR. The plot graph is augmented with technologies that provide input, output, and timed events at each state.

In our implementations of the conceptual model, we have found timed output events to be very useful. Instead of relying solely on a tight input-output scheme, we have introduced timers, which allow us to suspend the event to a later time and thereby give the author more control of the output. Fig. 4 illustrates the implementation of the CORRIDOR drama (discussed in more detail in Section 5). The primary input technology in the setup was Semacode tags placed in the streets of Aarhus and the primary output was audio played through the user's headset. 
However, a number of the Semacode tags also triggered a timer that would send a text message to the user's mobile phone at a later time. At the final state E, the user would also receive a timed phone call and receive audio from speakers in the environment (which in this case were placed on an actual theater stage). The figure also illustrates how real actors were part of the CORRIDOR drama. The users would meet the actors while walking from one location to another, however, the actors did not provide input or output in a system sense, but interacted directly with the user.

The final aspect of an implementation that needs consideration is how to bridge the drama with the real world scenography. Fortunately, each of the input technologies discussed above make it relatively easy to anchor the plot to real physical locations. A GPS sensor attached to the mobile phone, for instance, can provide precise physical coordinates for a location. The story can then be written to take the physical location into account, e.g. by telling the user to "walk to the other end of the market square" when the system has registered that the user has entered the GPS-coordinate for the square. 2D barcodes such as Semacodes cannot provide such a global position, but since the tags are placed at physical locations, it is still possible to map the story events to those locations, i.e., the GPS example may be implemented simply by placing a Semacode close to the market square providing the same message as before.

Finally, it should be noted that there is not necessarily a one-to-one correspondence between story events and physical locations. Indeed, the author may often wish to create a branch between different output events at a given physical location in order to provide differentiated output that depends on the state of the user model at that particular location. However, it is of course also possible to create a direct mapping, which is the case in the CORRIDOR implementation depicted in Figure 4, where each state refers to a particular physical location and incorporates this into the story.

\subsection{Implementation Used for the Three Dramas}

Section 2.2 discussed how the conceptual framework can be realized by utilizing a number of alternative technologies. Here we discuss the technical implementations we have made so far. The applications presented here are built on a common urban computing infrastructure that we have developed for a variety of applications [8].

The applications rely on Semacode tags as input to anchor the story fragments to physical locations. Audio, video, and text is used as output on the mobile phones.

The software running the dramas was implemented on the Java JME platform and deployed on SonyEricsson K800i mobile phones, which include good camera support for scanning the tags and the possibility for using normal stereo headphones, for quality audio output. As the dramas included hundreds of megabytes of audio files, these files were not downloaded from the Web over the mobile Internet, but preloaded on a number of mobile phones that users would borrow when buying tickets.

Each Semacode tag encoded a unique URL and since the application used a fixed number of tags, each encoded URL was linked to a specific audio file in the application's environment model. When a tag was scanned it would trigger the corresponding audio file or timer event. However, the URLs also pointed to the Webpage of theatre Katapult and if scanned by a third-party Semacode application, they would redirect the phone's browser to an information page describing the audio theater and explaining how to buy tickets. 


\section{Example Plays and Initial Experiences}

To date, the framework has been utilized in three Mobile Urban Dramas, "CORRIDOR", "GAMA - ON THE TRAIL OF UNKNOWN LAND" and the outdoor school project "HASLEINTERACTIVE", which will be launched this summer. The Danish theatre, Katapult [10], has developed all of the plays, which are described in the following.

\subsection{Corridor}

The first drama "CORRIDOR" was developed for the Aarhus Festival in 2007. The amount of scenes (tags) to be scanned was sixteen, and the duration of the play was approx. 1 hour and $40 \mathrm{~min}$. The user is staged as a female journalist who is assigned to make a cover story on a secret PR manager due the next day. However, the PR manager keeps changing location, and moreover, a personal matter becomes urgent. As the plot progresses, the stress level increases and the journalists' officious boss keeps phoning her. By walking in the streets of Aarhus, the user finds herself in a dramatised reality, where everyone could be a possible character in the play. Four actors and two actresses take part in the play, and moreover, the user receives events on the phone, that simulates SMS messages and calls. The staging of the Mobile Urban Drama is essential for the experience of the play and to whether or not the users can relate to the story, and accept the premise of being the main character. After having experienced the play, several users stated that they actually felt stressed out.

A total of more than 100 people experienced the play during the seven days it was staged last summer. Thirty-three of these completed an informal questionnaire consisting of open questions formulated by the theatre. On the question, "What is your overall experience of the play?" $94 \%$ answered, that they liked it and found it very exciting, and an interesting manner in which to experience the city.

On the question, "Was it difficult to navigate around the city?" $21 \%$ noted that the construction work in a particular part of the city made it more difficult, however, not impossible, to navigate. Furthermore, $13 \%$ would have preferred the ability to have the description repeated of which way to go; especially those users who did not know the city beforehand. However, most commented, that they found their way effortless. One commented on the map and another user on the ability to call for help on the service phone number as helpful features. On the question "Did you find it difficult to make use of the mobile phone - or to scan the tags?" $85 \%$ answered "No". However, $12 \%$ had to scan the tags more than once, and one user stated, that being stressed out with somewhat shaking hands made it more difficult to scan the tags.

In the last question, users were asked if they had any suggestions for improvement. $33 \%$ would have liked an increased sound volume as the noisy environment could result in them not being able to hear the play. $18 \%$ would have liked to meet more actors in the play, or a larger degree of interaction. Finally, 12\% suggested a larger degree of personal influence on the play - e.g. making choices. These results had an impact on the work on the second project, GAMA. 


\subsection{GAMA}

The second project "GAMA - ON THE TRAIL OF UNKNOWN LAND" is played in the city of Horsens, and will play throughout the summer of 2008. The play consists of 14 scenes (tags) and the duration is $1 \mathrm{~h}$. and $20 \mathrm{~m}$. The story is about a medical student, Christian, who is on his way to his sisters funeral. On his way to church, he receives a mysterious phone call from a woman who claims to have a message from his sister, Johanne. The woman claims to be Anna Christina Bering, the long-dead wife of a famous explorer, Vitus Bering, who was descended from Horsens. The user is the main character in a psychological treasure hunt; and he is provided with historical facts of the city through his quest for messages from his late sister.

The overriding conceptual structure is the same in CORRIDOR and GAMA; both plays follow the classical Hollywood model, which is a linear course where the drama increases until a climax at the end of the plot. In the GAMA play, the user is more interactive than in CORRIDOR, as the user needs to find clues, e.g. old letters in drawers and other objects in his quest. As opposed to CORRIDOR, in GAMA several scenes take place indoor, and thus making better use of the surrounding environment than in CORRIDOR. Another considerable difference in the two urban

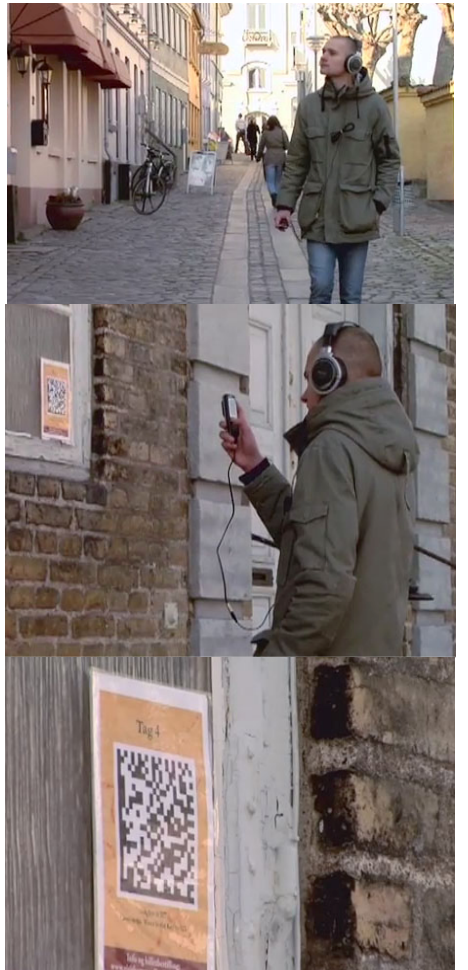

Fig. 5. The user photographs tags as he walks in the city, which enables him to listen to the audio drama dramas is the fact that in GAMA the locations were determined before the drama was written. This meant that the individual audio sequences were written accordingly to the approximate distance to the next location, which made less pauses in the storyline, and thus, the long music sequences that were utilized in CORRIDOR unnecessary. The duration of the longest audio clip in GAMA was eleven minutes, which has proven not to be too long - even to those users that walk quickly. Furthermore, the play could be made more place-specific when having determined the physical locations before the writing process. This makes the user experience more immersive, however, more difficult for the writers to compose having more restrictions than when dealing with classic theatrical dramas.

General experiences and lessons learned on urban dramas include not making the course through the city too complex. It should not be too difficult to find the way to the next tag as this would cause an increased focus on the map when navigating through the city, and less focus on the drama and the city itself. Furthermore, the tags should be carefully placed and protected. 


\subsection{HasleInteractive - Outdoor School}

HASLEINTERACTIVE is a play being launched in September 2008. Unlike CORRIDOR and GAMA, it is developed for educational purposes for public schools; more specifically, sixth graders. Moreover, the narrative consists of both individual and social courses in the same play, as the audio sequences are supplemented by group work on tasks with close ties to the narrative. The duration of the play will be approx. three hours.

The narrative is developed for the hilly landscape of Hasle in the outskirts of Aarhus. The plotline is set in the year 2022 when large scenic areas of nature are dying out. Two scientists need help from children - only they can reveal the reason for the decaying nature. Adults cannot enter infected areas without being lethally drained of energy. The scientists do not understand the mysterious codes and events in the area, thus they send in the children, whose mission is to analyse, decipher mysterious codes, report back and find evidence from the biotopes - before it is too late.

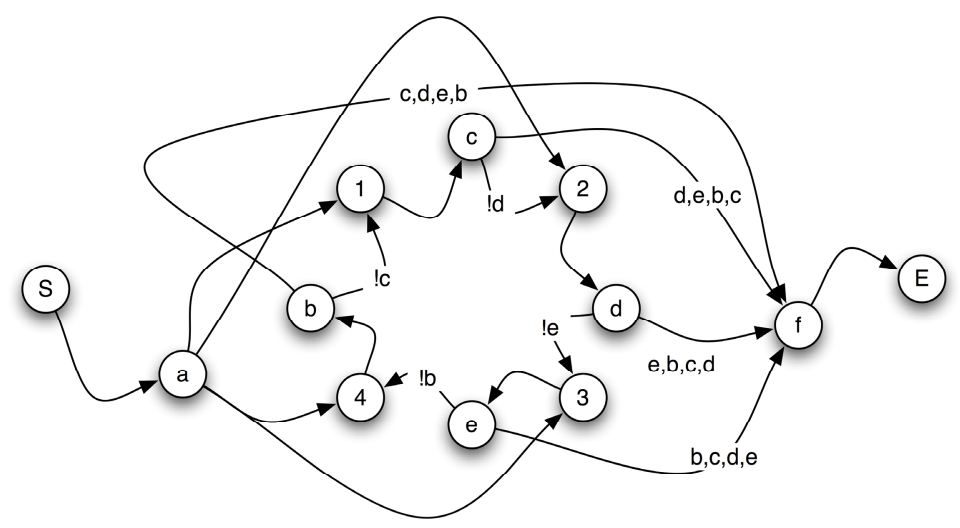

Fig. 6. An episodic plot graph from the HASLEINTERACTIVE drama

The aim for their learning is to gain an understanding of the correlation between humans and nature. In doing this, each group of pupils are to solve four assignments in the subjects mathematics and natural sciences at four checkpoints - two located in different lake biotopes, and two in a young forest and an old forest, respectively (number 1-4 in Figure 6). When the pupils arrive at a checkpoint, they scan a tag and receive assignments developed in collaboration with teachers. The amount of tags in the play is six when counting in the tags at the beginning and at the end of the play. However, between the checkpoints are "coordination nodes" with mysterious signs on the trees providing the pupils with more abstract info related to the narrative (nodes a-f in Figure 6). These nodes tie the narrative by providing a relationship between individual sensuous audio drama experiences and social technical experiences.

Figure 6 shows a plot graph from the HASLEINTERACTIVE drama. The guards limit the user's options and there are only four possible plots (given by the choice in node a). One of the reasons for creating the structure in Figure 6 is to allow more groups of users to use the same (physical) scenography at a time. Four different groups of threefour pupils may experience the play without interfering with each other except for the 
common introduction ( $\mathrm{S}, \mathrm{a})$ and end $(\mathrm{f}, \mathrm{E})$. The pupils do not begin at the same checkpoint - e.g. some start by walking to the lake, others to the forest.

Inspecting the graph reveals that the plots are made out of four self-sufficient episodes - the node pairs $(b, 1),(c, 2),(d, 3)$, and $(e, 4)$. The narrative arc of the play peaks for each node pair which makes the dramaturgical model an episodic wave model with four smaller climaxes. This narrative model makes it indifferent in which order the checkpoints are experienced, since their ordering depends on the path from node a. However, the narrative is written such that the order of the checkpoints and coordination nodes is fixed once the group is assigned a starting point (Figure 6).

At the end of the play, all of the pupils hear the same audio sequence. Though, the girls and boys get different versions of the same content of the audio files - that is, featuring a girl's voice for the girls, and a boy's voice for the boys. This is carried out in order to make it easier for the children to identify with the characters in the play. The theatre finds identification very important for the overall experience.

The incentive for combining dramatic courses and classes is to strengthen the learning possibilities by stimulating more senses; and to stage the learning in another and more exiting and motivating setting utilizing nature as the classroom.

Working with Mobile Urban Dramas for nature areas and not for cityscapes turned out not to be a limitation but rather an advantage. A forest or a lake can also be very dramatic settings; and when the children walk through the forest and hear the plaintively voices of the trees in the play, it is an advantage that the voices seem to emanate from one tree for one pupil, and from another tree for another pupil, depending on the position of the children.

\section{Related Work}

The three Mobile Urban Dramas developed with our framework represent a novel contribution to the domain of reality theatre/mobile drama. We briefly compare the Mobile Urban Dramas with state of the art. Earlier examples of urban storytelling include PodWalks [14] and AudioWalks [2], where the user downloads audio files to her mp3 player and listens to a narrative as she walks in the city. While these narratives refer to elements in the urban environment, there is no system support for integrating the city in the play's scenography and the stories are not interactive.

The "Call Cutta" [15] mobile phone play uses physical rooms in the city as scenography, and when the audience (a user) arrives, a phone rings and the play takes place as a partly improvised conversation between a real call centre person (actor) and the user. After a scene the user may continue to another room and have a new partly improvised conversation with another call centre actor. Compared to "Call Cutta" our concept provides a coherent storyline following a branching plot graph connecting a path through the city as the scenography. The user listens to the (branching) storyline while moving between the Semacode tagged locations that may involve some real actor activity, inspection of physical things, etc. in conjunction with a transition to a new audio scene in the headphones.

"Cargo Sofia" [4] has a storyline that develops relative to the route and locations visited, but the audience experience it only through a video projection shown in the dark container room, which resembles the traditional theatre arena, only smaller and 
driving around in the streets. Even though there are similarities to Mobile Urban Dramas, "Cargo Sofia" does not utilize the native city locations as scenography, the city is experienced only through a video feed on the wall, and there are no possibilities for individual (e.g. branching) experiences or an individual pace on the route.

"Water" [18] is categorized as augmented reality fiction. Equipped with a notebook, GPS-System and earphones, the user walks to explore a story, which is determined by their physical movement. Like the mobile urban drama, "Water" aims to create a strong relation between the story and he surroundings. However, "Water" only explores single input (GPS) and output (audio from a notebook) and not the generalised concept that mobile urban drama incorporates.

Song Fuer C [19] is probably the project that comes closest to our Mobile Urban Dramas, but there is to our knowledge no usage of location-based technology, that allow individual branching or individual paces on the routes coupled to the storyline.

The New York eRuv [7] path provides a similar Semacode tagged path but with more factual information linked to the tags, and no branching story line. Thus it boils down to a location-based audio guide.

\section{Conclusion and Future Work}

We have developed a mobile phone software framework for realizing a novel dramaturgical genre - Mobile Urban Drama. The framework and the manuscripts developed by Katapult have given rise to a new genre of pervasive theatre experiences taking advantage of the user as the main character and utilizing the urban environment as the scenography. The framework is currently in use for several plays that have had several hundreds of paying theatre visitors. The experiences are generally very positive both from the audience and theatre reviewers from magazines and TV. We wish to develop the concepts further and we are taking advantage of the real use experiences in the development of new elements to the play support.

These development plans include a general reconnaissance of what the possibilities are - technical as well as dramaturgical - when designing mobile urban dramas. Further, the plans include a larger degree of user influence; e.g. giving the users the opportunity to make more elaborate and detailed choices in the story line. Moreover, different types of sensors and actuators are being integrated in the environment to activate the physical surroundings of the play - such as the buildings and interior of the city - to become interactive. In this manner, the surroundings will to a greater extent become an active part of the play, e.g. by means of slamming doors, showers of water, ambient sounds etc. This may give the audience interactive experiences using the city as an interactive theatrical scenography.

Acknowledgements. We thank our colleagues at www.interactivespaces.net. We would also like to thank the theatre Katapult, in particular Torben Dahl, Eva Mikkelsen and Solrun Toft Iversen, as well as the writers of the three plays, Michael Monberg, Bjørn Rasmussen and Mia Brandstrup Andersen, respectively. Further, we would like to thank for the support from VisitHorsens and HasleInteractive. 


\section{References}

1. 4D-film (September-08-2008), http://en.wikipedia.org/wiki/4-D_film

2. AudioWalks (September-08-2008), http://www. flamingoeffekten.dk/\#41673

3. Bernstein, M.: Storyspace 1. In: Blustein, J. (ed.) Proceedings of the Thirteenth ACM Conference on Hypertext and Hypermedia, HYPERTEXT 2002, College Park, Maryland, USA, June 11 - 15, 2002, pp. 172-181. ACM, New York (2002)

4. Brady, S.: Dicking Cargo Sofia - A Bulgarian Truck Ride through Dublin. In: TDR (The Drama Review), vol. 51(4) (T196), pp. 162-167 (Winter 2007)

5. Brusilovsky, P.: User Modeling and User-Adapted Interaction. Adaptive hypermedia, Ten Year Anniversary Issue 11, 87-110 (2001)

6. Cavazza, M., Pizzi, D.: Narratology for Interactive Storytelling: A Critical Introduction. In: Göbel, S., Malkewitz, R., Iurgel, I. (eds.) TIDSE 2006. LNCS, vol. 4326, pp. 72-83. Springer, Heidelberg (2006)

7. eRUV. A Street History in Semacode (September-08-2008), http://www.dziga. com/eruv/index.php

8. Hansen, F.A., Grønbæk, K.: Social web applications in the city: a lightweight infrastructure for urban computing. In: Proceedings of the Nineteenth ACM Conference on Hypertext and Hypermedia, Pittsburgh, PA, USA, June 19 - 21, 2008, pp. 175-180 (2008)

9. Hansen, F.A., Bouvin, N.O., Christensen, B.G., Grønbæk, K., Pedersen, T.B., Gagach, J.: Integrating the Web and the World: Contextual Trails on the Move. In: Proceedings of the 15th ACM Hypertext 2004 Conference, Santa Cruz, USA (2004)

10. Katapult Theatre (only in Danish) (September-08-2008), http: / /www . katapult. dk /

11. Kobsa, A.: User modelling: Recent work, prospects and hazards. In: SchneiderHufschmidt, M., Kühme, T., Malinowski, U. (eds.) Adaptive User Interfaces: Principles and Practice, pp. 111-128. North-Holland, Amsterdam (1993)

12. Mateas, M., Stern, A.: Integrating plot, character and natural language processing in the interactive drama Façade. In: Gobel, S., et al. (eds.) Proceedings of the 1st Intl. Conference on Technologies for Interactive Digital Storytelling and Entertainment (TIDSE 2003), March 24-26. Fraunhofer IRB Verlag, Darmstadt (2003)

13. Mateas, M., Stern, A.: Façade, an experiment in building a fully-realized interactive drama. In: Game Developers Conference (GDC 2003), San Jose, CA, USA, March 4-8 (2003)

14. PodWalks (September-08-2008), http://www. cphx. dk/index . php?id=34124

15. Rimini-Protokoll (September 2008), http: / /www. rimini-protokoll. de/website/ en/project_2766.html

16. Röber, N., Huber, C., Hartmann, K., Feustel, M., Masuch, M.: Interactive Audiobooks: Combining Narratives with Game Elements. In: Göbel, S., Malkewitz, R., Iurgel, I. (eds.) TIDSE 2006. LNCS, vol. 4326, pp. 358-369. Springer, Heidelberg (2006)

17. Ryan, M.-L.: Avatars of Story. University of Minnesota Press, Minneapolis (2006)

18. Schemat, S.: Water (Augmented Reality Fiction) (2004) (September-08-2008), http: // www.kunstverein-cuxhaven.de/ohne_schnur/englisch/unterseiten/ schemat.htm

19. Song Fuer, C.: Homepage (September-08-2008), http: / / www . song fuerc . de/

20. Szilas, N.: Stepping into the Interactive Drama. In: Göbel, S., Spierling, U., Hoffmann, A., Iurgel, I., Schneider, O., Dechau, J., Feix, A. (eds.) TIDSE 2004. LNCS, vol. 3105, pp. 14-25. Springer, Heidelberg (2004) 